\title{
The origin of the enhanced oxygen storage capacity of $\mathrm{Ce}_{1-x}(\mathrm{Pd} / \mathrm{Pt})_{x} \mathrm{O}_{2}$
}

\author{
David O. Scanlon,* Benjamin J. Morgan $†$ and Graeme W. Watson* \\ Received 29th August 2010, Accepted 17th November 2010 \\ DOI: $10.1039 / \mathrm{c0cp01635g}$
}

Doping $\mathrm{CeO}_{2}$ with $\mathrm{Pd}$ or Pt increases the oxygen storage capacity (OSC) and catalytic activity of this environmentally important material. To date, however, an understanding of the mechanism underlying this improvement has been lacking. We present a density functional theory analysis of Pd- and Pt-doped $\mathrm{CeO}_{2}$, and demonstrate that the increased OSC is due to a large displacement of the dopant ions from the Ce lattice site. $\mathrm{Pd}(\mathrm{II}) / \mathrm{Pt}(\mathrm{II})$ (in a $d^{8}$ configuration) moves by $\sim 1.2 \AA$ to adopt a square-planar coordination due to crystal field effects. This leaves three three-coordinate oxygen atoms that are easier to remove, and which are the source of the increased OSC. These results highlight the importance of rationalizing the preferred coordination environments of both dopants and host cations when choosing suitable dopants for next generation catalysts.

\section{Introduction}

$\mathrm{CeO}_{2}$ is one of the key materials in modern three-way catalysts, where it has been highly effective in the catalysis of automotive emissions, both as a support and as a catalyst itself. ${ }^{1-3}$ This material also finds applications in other catalytic processes - e.g. water-gas shift reaction, and $\mathrm{SO}_{x}$ reductionas well as being a component in gas sensors, intermediate temperature solid oxide fuel cells, and field-effect transistors. ${ }^{4}$ The importance of ceria as a catalyst and a support stems from its oxygen storage capacity (OSC), which allows it to release oxygen under reducing conditions and to store oxygen by filling oxygen vacancies under oxidizing conditions. ${ }^{5}$ Increasing the number and mobility of oxygen vacancies enhances the OSC and correspondingly improves catalytic activity. ${ }^{6,7}$

In this increasingly environmentally conscious age the search for improved catalysts has become inexorable. To this end, the doping of $\mathrm{CeO}_{2}$ to produce next-generation catalysts has gathered much attention. In three-way catalysts noble metals normally sit atop the $\mathrm{CeO}_{2}$ support. Recently, however, substitution of noble metals for $\mathrm{Ce}$ at lattice sites has been identified as a promising route to improved catalysts, and has been termed "noble metal ionic catalysis". 8

$\mathrm{Ce}_{1-x} \mathrm{NM}_{x} \mathrm{O}_{2-\gamma}$ solid solutions $(x=0.01-0.02)$, with $\mathrm{NM}=\mathrm{Cu}^{\mathrm{II}}, \mathrm{Pd}^{\mathrm{II}}, \mathrm{Pt}^{\mathrm{II}}$, and $\mathrm{Rh}^{\mathrm{III}}$, have all been previously considered. ${ }^{9-12}$ The presence of Pd in the lattice of ceria has been shown to have a beneficial catalytic effect for methane combustion, ${ }^{13}$ and allows higher rates of $\mathrm{NO}$ and $\mathrm{N}_{2} \mathrm{O}$ reduction, ${ }^{14}$ and higher rates and lower activation energies for the oxidation

School of Chemistry, Trinity College Dublin, Dublin 2, Ireland.

E-mail: scanloda@tcd.ie,watsong@tcd.ie

$\dagger$ Current address: Department of Materials, University of Oxford,

Parks Road, OX1 3PH, UK. of $\mathrm{CO}$ and hydrocarbons. ${ }^{9,14}$ Pt-doped $\mathrm{CeO}_{2}$ has demonstrated enhanced catalytic activity towards $\mathrm{CO}$ oxidation, hydrocarbon oxidation, and hydrogen adsorption, compared to that of $\mathrm{Pt}$ metal over $\mathrm{CeO}_{2} .{ }^{15} \mathrm{Pt}$-doped $\mathrm{CeO}_{2}$ was also reported to catalyze the gas-phase hydrogenation of benzene to cyclohexane, yielding a turnover frequency an order of magnitude higher than for $\mathrm{Pt}$ metal, $\mathrm{Pt} / \alpha-\mathrm{Al}_{2} \mathrm{O}_{3}$, or $\mathrm{Pt} / \gamma-\mathrm{Al}_{2} \mathrm{O}_{3} .{ }^{16}$ The presence of these lower valence dopant ions results in the formation of charge-compensating oxygen vacancies neighbouring the dopant, which are observed in extended X-ray absorption fine structure (EXAFS) experiments. ${ }^{9,17,18}$ For $\mathrm{Pd}$ and $\mathrm{Pt}$, doping is formally described as

$$
\mathrm{NM}_{(s)}+\mathrm{Ce}_{\mathrm{Ce}}^{\times}+\mathrm{O}_{\mathrm{O}}^{\times} \rightarrow \mathrm{NM}_{\mathrm{Ce}}^{\prime \prime}+\mathrm{V}_{\mathrm{O}}^{\bullet \bullet}+\mathrm{Ce}_{(s)} .
$$

It should be emphasised that the vacancy formation that accompanies the substitution of $\mathrm{Ce}^{\mathrm{IV}}$ with $\mathrm{NM}^{\mathrm{II}}$ is not a reduction that contributes to the OSC, but is a consequence of the system maintaining charge neutrality as part of the doping process.

Hermansson and co-workers have previously used the local density approximation (LDA) $+U$ approach to model the doping of $\mathrm{CeO}_{2}$ with $\mathrm{Pd}$ and $\mathrm{Pt},{ }^{19,20}$ with the formation of the charge-compensating vacancy incorrectly considered as the reduction energy. The authors reported that the replacement of a $\mathrm{Ce}^{\mathrm{IV}}$ in both cases resulted in a very small relaxation around the dopants. ${ }^{19,20}$ Subsequent removal of an oxygen neighbouring the dopant yielded "reduction" energies of $57 \mathrm{~kJ} \mathrm{~mol}^{-1}$ and $93 \mathrm{~kJ} \mathrm{~mol}^{-1}$ for Pd-doped and Pt-doped ceria, respectively. These results would suggest that the removal of an oxygen next to the dopant during formation of a charge-compensating vacancy costs energy. ${ }^{19,20}$ This is at variance with previous X-ray photoelectron spectroscopy studies that indicate $\mathrm{Pd}$ and $\mathrm{Pt}$ to be present preferentially in 
(a)

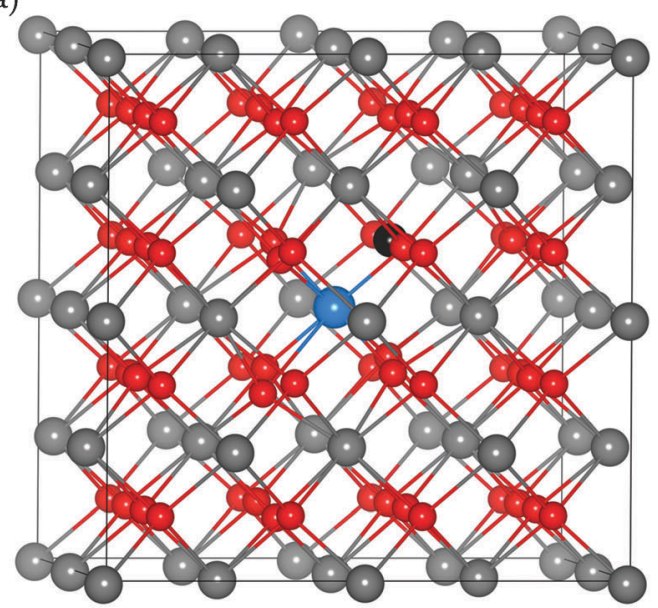

(b)

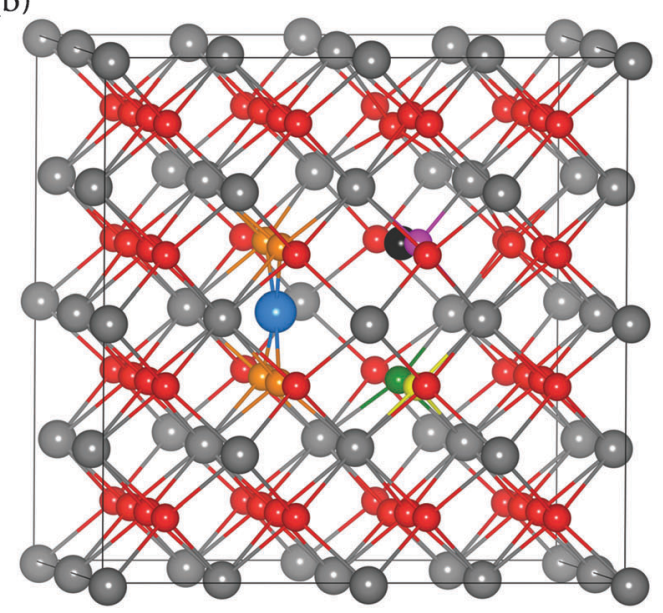

Fig. 1 (a) $\mathrm{Pd} / \mathrm{Pt}$ dopant in $\mathrm{CeO}_{2}$ sitting on the perfect $\mathrm{Ce}$ lattice position. (b) $\mathrm{Pd} / \mathrm{Pt}$ sitting in a square planar coordination between four lattice oxygen. Blue spheres denote the $\mathrm{Pd} / \mathrm{Pt}$ dopant, grey and red spheres denote the $\mathrm{Ce}$ and $\mathrm{O}$ ions respectively. The black spheres represent the position of the charge-compensating vacancy, and the pink, yellow and green spheres represent the under-coordinated oxygen ions $\mathrm{O}_{a}, \mathrm{O}_{\mathrm{b}}, \mathrm{O}_{\mathrm{c}}$ respectively.

the +2 oxidation state, and accompanied by spontaneously formed charge-compensating vacancies. ${ }^{9,18}$

Despite the reported improvements in catalytic activity and OSC, the underlying reason for these has remained unclear. In this article we study the effect of $\mathrm{Pd}$ and $\mathrm{Pt}$ substitution in $\mathrm{CeO}_{2}$, focusing on the oxidation state and geometry of the dopants, and their effect on oxygen vacancy formation. Using the insights gained from this analysis, we propose guidelines for the rational design of next generation catalysts.

\section{Theoretical methods}

All calculations were performed using the VASP package, ${ }^{21,22}$ in which the valence electronic states are expanded within a plane-wave basis. The valence-core interaction is described using the projector augmented wave (PAW) approach, ${ }^{23}$ and cores of [He] for oxygen, and [Xe] for cerium are used. The Perdew-Burke-Ernzerhof generalized gradient approximation (GGA) exchange-correlation functional was used, ${ }^{24}$ and supplemented by a Dudarev $+U$ term. $^{25}$ Standard density functional calculations are unable to correctly describe the electronic structure of reduced $\mathrm{CeO}_{2},{ }^{26,27}$ due to the selfinteraction error inherent to such functionals, and which is acute for strongly localised orbitals such as transition metal $d$ or rare earth $f$ states. $^{28-30}$ The inclusion of a $+U$ correction allows this deficiency to be addressed without an excessive increase in the computational cost of the calculations. The sensitivity of results to the value of $U$ for GGA $+U$ calculations of ceria have previously been described, ${ }^{26,27}$ with a value of $U=5 \mathrm{eV}$ selected for the Ce $\mathrm{f}$ orbitals. The suitability of this value of $U$ has subsequently been verified by other studies, ${ }^{31,32}$ and has been used many times to successfully model reduced $\mathrm{CeO}_{2}$ systems. ${ }^{33-36} \mathrm{GGA}+U$ has yielded results in excellent quantitative agreement with hybrid DFT approaches. ${ }^{37-40}$ No $+U$ term was applied to the dopant states, as the $+U$ is used in this case as an energetic penalty to partial occupation, meaning that localized behaviour is favoured over delocalized behaviour. As there is only one dopant in the supercell, if charge wished to localize on the dopant, there will be no delocalization issue. A cutoff of $500 \mathrm{eV}$ was used for the all calculations, with the Brillouin zone sampled using a $4 \times 4 \times 4$ Monkhorst-Pack grid for the bulk and a $2 \times 2 \times 2$ sampling for the 96 atom supercell. All calculations were spin polarized and were deemed to have converged when the forces on all the atoms were less than $0.01 \mathrm{eV}^{-1}$.

\section{Results}

$\mathrm{CeO}_{2}$ is a wide band gap insulator that adopts a cubic fluorite structure with a lattice parameter, $a$, of 5.41. The calculated lattice parameter of $\mathrm{CeO}_{2}$ is $\sim 5.49 \AA$ at the $\mathrm{GGA}+U ; U=5 \mathrm{eV}$ level, which is within $1.5 \%$ of experiment. Using a $2 \times 2 \times 2$ (96 atom) supercell, we have calculated the formation energy of an oxygen vacancy in pure $\mathrm{CeO}_{2}$ to be $253 \mathrm{~kJ} \mathrm{~mol}^{-1}$, which is consistent with previous theoretical studies. ${ }^{41}$

For the incorporation of $\mathrm{Pd} / \mathrm{Pt}$ into the $\mathrm{CeO}_{2}$ lattice we first considered the case of single $\mathrm{Pd} / \mathrm{Pt}$ substituting for a $\mathrm{Ce}^{\mathrm{IV}}$ within the 96 atom cell, with subsequent charge-compensating vacancy formation. This was done in two ways: (i) similar to the calculations of Hermansson and co-workers, we replaced one $\mathrm{Ce}^{\mathrm{IV}}$ with a $\mathrm{Pd} / \mathrm{Pt}$ on the perfect lattice site, and allow this to relax fully, followed by the subsequent removal of an oxygen neighbouring the dopant (charge-compensating vacancy formation, Fig. 1(a)) and (ii) the $\mathrm{Pd} / \mathrm{Pt}$ is displaced off the perfect $\mathrm{Ce}$ lattice site upon charge-compensating vacancy formation, to test for lower energy distorted configurations.

From method (i), the energy to form a charge-compensating vacancy in $\mathrm{Pd} / \mathrm{Pt}$ doped $\mathrm{CeO}_{2}$ was found to be $64 \mathrm{~kJ} \mathrm{~mol}^{-1}$ and $115 \mathrm{~kJ} \mathrm{~mol}^{-1}$ for $\mathrm{Pd}$ and $\mathrm{Pt}$, respectively, which is in reasonable agreement with the previous $\mathrm{LDA}+U$ results of Hermansson and co-workers. ${ }^{19,20}$ From method (ii), however, once the $\mathrm{Pd} / \mathrm{Pt}$ is allowed to move off the perfect Ce lattice site, it undergoes a large lattice distortion away from the charge-compensating vacancy site to assume a square planar coordination between four lattice oxygens, Fig. 1(b). The charge-compensating vacancy formation energies in this case 
are now $-69 \mathrm{~kJ} \mathrm{~mol}^{-1}$ and $-72 \mathrm{~kJ} \mathrm{~mol}^{-1}$ for $\mathrm{Pd}$ and $\mathrm{Pt}$, respectively.

The large lattice distortion lowers the energy of the square planar configuration compared to the distorted cubic configuration proposed previously ${ }^{19,20}$ by $134 \mathrm{~kJ} \mathrm{~mol}^{-1}$ and $186 \mathrm{~kJ} \mathrm{~mol}^{-1}$ for $\mathrm{Pd}$ and $\mathrm{Pt}$, respectively. Indeed, with a charge-compensating vacancy present, $\mathrm{Pd} / \mathrm{Pt}$ will have an oxidation state of +2 with a $d^{8}$ configuration, which is not known to adopt the cubic coordination of the perfect $\mathrm{Ce}$ lattice position. ${ }^{42}$ Our results indicates that the $\mathrm{NM}^{\mathrm{IV}}$ to $\mathrm{NM}^{\mathrm{II}}$ plus charge-compensating vacancy transition is in fact spontaneous, which is consistent with experiment, with a charge-compensating vacancy expected to be neighbouring the dopant. ${ }^{9,17,18}$ The failure to account for the large lattice distortion explains why Hermansson and co-workers predicted the incorporation of the noble metal at a higher oxidation state, and the formation of the charge-compensating vacancy to be a reduction. It is also instructive to note that, in supercell defect calculations, breaking of the symmetry has been shown to be vital to obtain quantitatively accurate defect geometries. $^{43,44}$

The large lattice distortion places the $\mathrm{Pd} / \mathrm{Pt}$ in a square planar coordination environment between 4 lattice oxygens, and leaves three under-coordinated oxygen atoms, Fig. 1(b). Table 1 lists calculated formation energies for vacancies at each of the three under-coordinated oxygen. The oxygen vacancy formation energy decreases from $253 \mathrm{~kJ} \mathrm{~mol}^{-1}$ in pure $\mathrm{CeO}_{2}$ to $173 \mathrm{~kJ} \mathrm{~mol}^{-1}$ for Pd-doped and $170 \mathrm{~kJ} \mathrm{~mol}^{-1}$ for Pt-doped $\mathrm{CeO}_{2}$. For all three under-coordinated oxygen atoms, the energy to form an oxygen vacancy is significantly lowered compared to pure $\mathrm{CeO}_{2}$. For both dopants, the undercoordinated oxygen that is furthest from the charge-compensating vacancy (denoted $\mathrm{O}_{\mathrm{b}}$, yellow sphere in Fig. 1(b)), is the easiest to remove, as this minimizes the repulsion between the two positively charged vacancies. The other under-coordinated oxygen ions $\left(\mathrm{O}_{\mathrm{a}}\right.$ and $\left.\mathrm{O}_{c}\right)$ have a higher vacancy formation energy, but are still easier to remove than an oxygen from pure $\mathrm{CeO}_{2}$.

The excess electrons left behind upon vacancy formation localize on two Ce ions neighbouring the vacancy, formally reducing them to $\mathrm{Ce}^{\mathrm{III}}$, as illustrated in Fig. 2. The lowering of the oxygen vacancy formation energy in the doped supercell compared to the pure supercell indicates that the large lattice distortion serves to weaken the bonds around the under-coordinated oxygen, making it easier to remove them. In the work of Hermansson and co-workers, the $\mathrm{Pd} / \mathrm{Pt}$ states in the band gap are proposed as the origin of the enhanced reducibility. ${ }^{19,20}$ As their study does not find the lowest energy square planar configuration, and ignores the formation of a charge compensating vacancy, the author's calculated PEDOS for doped $\mathrm{CeO}_{2}$ shows $\mathrm{Pd} / \mathrm{Pd}$ defect states in the band gap, with

Table 1 GGA $+U$ calculated oxygen vacancy formation energies for the three under-coordinated oxygens in $\mathrm{Pd} / \mathrm{Pt}$ doped $\mathrm{CeO}_{2}$ supercell, $\mathrm{O}_{\mathrm{a}}, \mathrm{O}_{\mathrm{b}}, \mathrm{O}_{\mathrm{c}}$. All energies are quoted in $\mathrm{kJ} \mathrm{mol}^{-1}$

\begin{tabular}{llll}
\hline & $V_{\mathrm{O}_{\mathrm{a}}}$ & $V_{\mathrm{O}_{\mathrm{b}}}$ & $V_{\mathrm{O}_{\mathrm{c}}}$ \\
\hline $\mathrm{CeO}_{2}: \mathrm{Pd}$ & 224 & 173 & 228 \\
$\mathrm{CeO}_{2}: \mathrm{Pt}$ & 207 & 170 & 218 \\
\hline
\end{tabular}

some unoccupied defect states in the spin down (Beta spin). ${ }^{19,20}$ Subsequent oxygen vacancy formation fills these unoccupied states, leaving the non-spin paired $\mathrm{Pd} / \mathrm{Pt}$ states in the band gap. ${ }^{19,20}$

In our study, we have uncovered the ground state structure of $\mathrm{Pd} / \mathrm{Pt}$ doped $\mathrm{CeO}_{2}$, which is in the square planar coordination with a charge compensating vacancy neighbouring the dopant. In a square planar $d^{8}$ configuration, the electrons spin pair, as can be seen in our calculated PEDOS for Pd doped $\mathrm{CeO}_{2}$, Fig. 3(a). Upon reduction of the system (removal of an oxygen vacancy), the excess electrons localize on the two neighbouring Ce atoms. This is clearly shown in the PEDOS of the reduced Pd doped supercell, Fig. 3(b). Therefore, our study shows that the large lattice distortion and the reducibility of $\mathrm{Ce}^{\mathrm{IV}}$ to $\mathrm{Ce}^{\mathrm{III}}$ are the key factors in the lower reduction energy of $\mathrm{Pd} / \mathrm{Pt}$ doped $\mathrm{CeO}_{2}$.

Ligand field theory predicts that a $d^{8}$ ion will prefer a square planar coordination over that of a cubic coordination. Within a cubic crystal field the $d^{8}$ cation will have two paired electrons and two unpaired electrons in three high energy $t_{2 g}$ orbitals, Fig. 4. This degeneracy can be lifted by adopting a square planar arrangement, and the now doubly occupied $d_{x y}$ and $d_{z^{2}}$ orbitals are lowered in energy, stabilizing the ion, Fig. $4 .^{42}$ It is interesting to consider whether this crystal field preference for square planar coordination for the $d^{8}$ dopants is sufficient to induce the large lattice distortion, or whether the chargecompensating vacancy formation that accompanies such doping is necessary. To address this we have simulated the effect of a single $N^{\mathrm{II}}$ in the 96 atom cell with no vacancy present by adding two electrons to the system. We tested the stability and spin state of two configurations: (i) $\mathrm{Pd}^{\mathrm{II}} / \mathrm{Pt}^{\mathrm{II}}$ on the perfect cubic Ce lattice site, and (ii) $\mathrm{Pd}^{\mathrm{II}} / \mathrm{Pt}^{\mathrm{II}}$ in square planar arrangement between four lattice oxygens. The square planar configuration was $133 \mathrm{~kJ} \mathrm{~mol}^{-1}$ and $238 \mathrm{~kJ} \mathrm{~mol}^{-1}$, more stable than the cubic for $\mathrm{Pd}$ and $\mathrm{Pt}$, respectively. It is clear that even in the absence of a charge-compensating vacancy, $\mathrm{Pd}^{\mathrm{II}}$ and $\mathrm{Pt}^{\mathrm{II}}$ both strongly favour square planar coordination. The well known preference for these metals to adopt square planar coordination ${ }^{42}$ is sufficient to drive the large lattice distortion in doped $\mathrm{CeO}_{2}$.

\section{Discussion}

Noble metal ionic catalysis has emerged over the past decade as a viable alternative to the well established metal oxide catalysts. ${ }^{4-47}$ Despite multiple reports of improved catalytic behaviour of these noble-metal-doped oxides over pure oxide systems and even over metals supported on metal oxides, little is actually known about the true effect of these noble metal dopants on reducibility and catalytic selectivity/activity. To aid in progressing the development of noble metal ionic catalysis it is vital that a nanoscopic understanding of the fundamental role of the dopant in the host oxide is elucidated.

Our calculations have revealed that the driving force for enhanced reducibility in $\mathrm{Pd} / \mathrm{Pt}$ doped $\mathrm{CeO}_{2}$ is the preference for square planar coordination displayed by $d^{8} \mathrm{Pd}^{\mathrm{II}} / \mathrm{Pt}^{\mathrm{II}}$. Both $\mathrm{Pt}$ and $\mathrm{Pd}$ prefer to be incorporated into the $\mathrm{CeO}_{2}$ lattice as $\mathrm{Pd}^{\mathrm{II}} / \mathrm{Pt}^{\mathrm{II}}$, with an associated charge-compensating oxygen vacancy, and not as $d^{6} \mathrm{Pd}^{\mathrm{IV}} / \mathrm{Pt}^{\mathrm{IV}}$. The driving force for the 


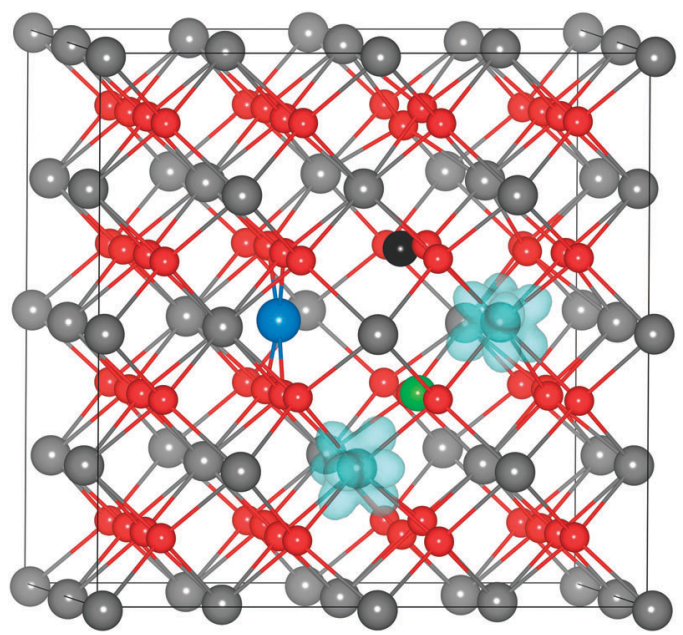

Fig. 2 Geometry and spin density of Pt-doped $\mathrm{CeO}_{2}$, with a vacancy in the $\mathrm{O}_{\mathrm{b}}$ position. The blue, grey, red, black, and green spheres represent the $\mathrm{Pt}, \mathrm{Ce}, \mathrm{O}$, charge compensating vacancy, and "reduction" vacancy respectively. The light blue spin density isosurface is shown

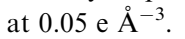
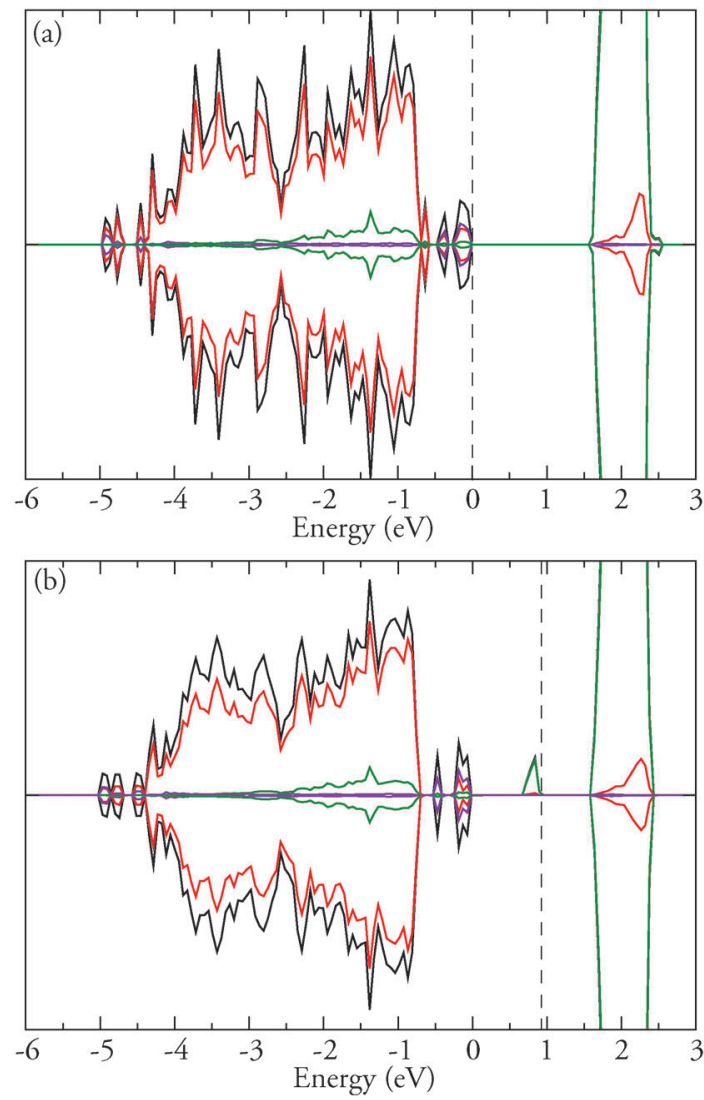

Fig. 3 Atom projected electronic densities of states for (a) Pd doped $\mathrm{CeO}_{2}$ with a charge compensating vacancy, and (b) reduced Pd doped $\mathrm{CeO}_{2}$ with a charge compensating vacancy. $\mathrm{O} p$ states are red, $\mathrm{Ce} f$ states are green and Pd $d$ states are violet. The top of the valence band is aligned to $0 \mathrm{eV}$ with the vertical dashed line showing the position of the highest occupied state.

large lattice distortion is the strong preference of $\mathrm{Pd}^{\mathrm{II}} / \mathrm{Pt}^{\mathrm{II}}$ to obtain square planar coordination environments. This large

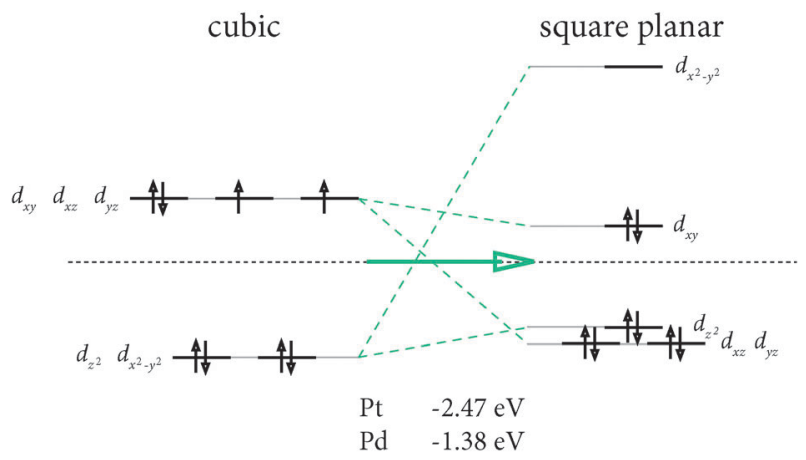

Fig. 4 Crystal field splitting of a $d^{8}$ ion in a cubic (left) and square planar (right) field, with the associated stabilization energies.

lattice distortion is determined by the electronic configuration of the noble metal dopant, and not purely by the valence of the dopant. To test this hypothesis, we carried out test calculations of $\mathrm{Zn}$ doped $\mathrm{CeO}_{2}$, finding that $d^{10} \mathrm{Zn}^{\text {II }}$ ions do not wish to adopt square planar coordination, or to remain on the cubic Ce lattice site, but rather prefer to distort to form 4 short and 4 long $\mathrm{Zn}-\mathrm{O}$ bonds. It is not surprising that $\mathrm{Zn}$ does not take up a square planar configuration as $\mathrm{Zn}$ prefers a tetrahedral coordination, as seen in $\mathrm{ZnO}{ }^{48}$ The electronic configuration and size of a dopant is thus of vital importance to the type of distortion that can be induced in the host oxide.

Normally, when one considers a suitable dopant to enhance the reducibility of a material, the main focus is placed on the ionic radius of the dopant, in an effort to distort the coordination of a lattice oxygen and weaken some of the $\mathrm{M}-\mathrm{O}$ bonds. ${ }^{49-51} \mathrm{It}$ is generally accepted that, for example, the increase in OSC of $\mathrm{Zr}$ and $\mathrm{Ti}$ doped $\mathrm{CeO}_{2}$ can be explained by the weakening of oxygen bonds around the dopant ion, ${ }^{50,51}$ with a recent study highlighting the role of relaxation around the dopant in $\mathrm{Zr}$ doped $\mathrm{CeO}_{2} .{ }^{52}$ Our calculations indicate that another significant factor is the preferred coordination environment of the dopant, especially in the case of $d$ block transition metals. Careful choice of a dopant with a suitably small ionic radius, and a significant difference in preferred coordination environment compared to the host cation, will lead to maximum effect on the oxygen bonding. These considerations suggest it is advantageous to choose a dopant with a normal coordination environment in its own native oxide that is lower than the coordination number in the host oxide, to maximize the distortion, and yield a reduced coordination around some of the oxygen ions. Hence, a careful consideration of the coordination "requirements" of the dopant in the host oxide could be exploited in the prediction in developing new improved metal oxide catalysts. It is therefore likely that other $d^{8}$ dopants, such as $\mathrm{Ni}^{\mathrm{II}}$, or even a $d^{9}$ dopant, such as $\mathrm{Cu}^{\mathrm{II}}$, would be excellent dopants to test these criteria.

The consequences of these electronic structure driven distortions extend beyond the OSC of $\mathrm{CeO}_{2}$. In the field of dilute magnetic semiconductors (DMS), doping of oxide semiconductors with magnetic cations to induce ferromagnetism is of huge interest. ${ }^{48} \mathrm{In}$ some cases, e.g. $\mathrm{ZnO}: \mathrm{Co}$, the coordination environments of the dopant (tetrahedral $\mathrm{Co}^{\mathrm{II}}$ in $\mathrm{Co}_{3} \mathrm{O}_{4}$ ) and host cation (tetrahedral $\mathrm{Zn}^{\mathrm{II}}$ in $\mathrm{ZnO}$ ) match and are not an issue ${ }^{48}$ however, in other cases the coordination of the ion will 
have a large impact on the splitting of the $d$ levels. A good example of this is $\mathrm{CeO}_{2}$ : $\mathrm{Co}$, which has been reported to show room temperature ferromagnetism. ${ }^{53,54}$ This has yet to be satisfactorily explained using first principles, and to date, all first principles calculations have assumed the Co to sit on the perfect Ce lattice cite. ${ }^{55,56}$ It would appear unlikely, based on our current analysis for $\mathrm{Pd} / \mathrm{Pt}$, that a $d^{7}\left(\mathrm{Co}^{\mathrm{II}}\right)$ or a $d^{6}$ $\left(\mathrm{Co}^{\mathrm{III}}\right)$ ion would prefer to sit on a perfect lattice site.

\section{Conclusion}

The origin of the improved OSC of $\mathrm{Pd} / \mathrm{Pt}$-doped $\mathrm{CeO}_{2}$ has been shown to be a consequence of the crystal field stabilization of the $d^{8}$ dopant ions in a square planar coordination environment. This requires the dopant $\mathrm{Pd}^{\mathrm{II}} / \mathrm{Pt}^{\mathrm{II}}$ to undergo a large lattice distortion away from the perfect $\mathrm{Ce}^{\mathrm{IV}}$ lattice site to assume a square planar coordination with four lattice oxygens. This large lattice distortion leaves three oxygen atoms under-coordinated in the vicinity of the dopant, making their removal under reducing conditions much more facile. These findings highlight the importance of the preferred coordination environment of the dopant compared with the cation coordination of the host oxide. We propose that when designing next-generation oxide catalysts with increased OSC, it is advantageous to select dopants based on an understanding of oxidation state, ionic radius, and additionally electronic structure and corresponding preferred coordination environments.

\section{Acknowledgements}

This work was supported by Science Foundation Ireland through the Research Frontiers Programme (grant numbers 08/RFP/MTR1044 and 09/RFP/MTR2274). Calculations were performed on the IITAC and Lonsdale supercomputers as maintained by TCHPC, and the Stokes supercomputer as maintained by ICHEC.

\section{References}

1 A. Trovarelli, Catalysis by Ceria and Related Materials, Imperial College Press, UK, 2002.

2 D. O. Scanlon, N. M. Galea, B. J. Morgan and G. W. Watson, J. Phys. Chem. C, 2009, 113, 11095-11103.

3 S. Fabris, S. de Gironcoli, S. Baroni, G. Vicario and B. Gabriele, Phys. Rev. B: Condens. Matter Mater. Phys., 2005, 71, $041102(\mathrm{R})$.

4 C. Loschen, A. Migani, S. T. Bromley, F. Illas and K. M. Neyman, Phys. Chem. Chem. Phys., 2008, 10, 5730-5738.

5 A. Trovarelli, Comments Inorg. Chem., 1999, 20, 263-284.

6 M. Daturi, N. Bion, J. Saussey, J.-C. Lavalley, C. Hedouin, T. Seguelong and G. Blanchard, Phys. Chem. Chem. Phys., 2001, 3, 252-255.

7 N. V. Skorodumova, S. I. Simak, B. I. Lundqvist, I. A. Abrikosov and B. Johansson, Phys. Rev. Lett., 2002, 89, 166601.

8 M. S. Hegde, G. Madras and K. C. Patil, Acc. Chem. Res., 2009, 42, 704-712.

9 K. R. Priolkar, P. Bera, P. R. Sarode, M. Hegde, S. Emura, R. Kamashiro and N. P. Lalla, Chem. Mater., 2002, 14, 2120-2128.

10 P. Bera, K. R. Priolkar, P. R. Sarode, M. S. Hegde, S. Emura, R. Kumashiro, V. Jayaram and N. P. Lalla, Chem. Mater., 2002, 14, 3591-3601.

11 P. Bera, K. R. Priolkar, A. Gayen, P. R. Sarode, M. S. Hegde, S. Emura, R. Kumashiro, V. Jayaram and G. N. Subbanna, Chem. Mater., 2003, 15, 2049-2060.
12 A. Gayen, K. R. Priolkar, P. R. Sarode, V. Jayaram, M. S. Hegde, V. Jayaram and S. Emura, Chem. Mater., 2004, 16, 2317.

13 A. Primavera, A. Trovarelli, C. de Leitenburg, G. Dolcetti and J. Llorca, Stud. Surf. Sci. Catal., 1998, 119, 87-92.

14 S. Roy, A. Marimuthu, M. S. Hegde and G. Madras, Appl. Catal., $B, 2007,71,23-31$.

15 T. Baidya, A. Gayen, M. S. Hegde, N. Ravishankar and D. L, J. Phys. Chem. B, 2006, 110, 5262-5272.

16 K. Nagaveni, G. Sivalingam, A. Gayen, A. Madras and M. S. Hegde, Catal. Lett., 2003, 88, 73-81.

17 P. Bera, A. Gayen, M. S. Hegde, N. P. Lalla, L. Spadero, F. Frusteri and F. J. Arena, J. Phys. Chem. B, 2003, 107, $6122-6130$.

18 A. Gayen, T. Baidya, A. S. Prakash, N. Ravishankar and M. S. Hegde, Indian J. Chem. A, 2005, 44A, 34-48.

19 Z. Yang, G. Luo, Z. Lu and K. Hermansson, J. Chem. Phys., 2007, 127, 074704

20 Z. Yang, G. Lu, Z. Lu, T. K. Woo and K. Hermansson, J. Phys.. Condens. Matter, 2008, 20, 035210.

21 G. Kresse and J. Hafner, Phys. Rev. B: Condens. Matter, 1994, 49, 14251-14271.

22 G. Kresse and J. Furthmuller, Comput. Mater. Sci., 1996, 6, 15-50.

23 P. E. Blöchl, Phys. Rev. B: Condens. Matter, 1994, 50, 17953.

24 J. P. Perdew, K. Burke and M. Ernzerhof, Phys. Rev. Lett., 1996, 77, 3865

25 S. L. Dudarev, G. A. Botton, S. Y. Savrasov, C. J. Humphreys and A. P. Sutton, Phys. Rev. B: Condens. Matter Mater. Phys., 1998, 57, 1505.

26 M. Nolan, S. Grigoleit, D. C. Sayle, S. C. Parker and G. W. Watson, Surf. Sci., 2005, 576, 217-229.

27 M. Nolan, S. C. Parker and G. W. Watson, Surf. Sci., 2005, 595(1-3), 223-232.

28 B. J. Morgan and G. W. Watson, Surf. Sci., 2007, 601, 5034-5041.

29 D. O. Scanlon, A. Walsh, B. J. Morgan and G. W. Watson, J. Phys. Chem. C, 2008, 112, 9903-9911.

30 B. J. Morgan, D. O. Scanlon and G. W. Watson, J. Mater. Chem., $2009,19,5175-5178$.

31 D. A. Andersson, S. I. Simak, B. Johansson, I. A. Abrikosov and N. V. Skorodumova, Phys. Rev. B: Condens. Matter Mater. Phys., 2007, 75, 035109.

32 C. Zhang, A. Michaelides, D. A. King and S. J. Jenkins, Phys. Rev. B: Condens. Matter Mater. Phys., 2009, 79, 075433.

33 M. Nolan, S. C. Parker and G. W. Watson, Phys. Chem. Chem. Phys., 2006, 8, 216.

34 M. Nolan and G. W. Watson, J. Phys. Chem. B, 2006, 110, 16600-16606.

35 M. Nolan, S. C. Parker and G. W. Watson, J. Phys. Chem. B, 2006, 110, 2256.

36 N. M. Galea, D. O. Scanlon, B. J. Morgan and G. W. Watson, Mol. Simul., 2009, 35, 577-583.

37 M. V. Ganduglia-Piravano, J. L. F. Da Silva and J. Sauer, Phys. Rev. Lett., 2009, 102, 026101.

38 H. Y. Li, H. F. Wang, Z. Q. Gong, Y. L. Guo, Y. Giuo, G. Lu and P. Hu, Phys. Rev. B: Condens. Matter Mater. Phys., 2009, 79, 193401.

39 J. L. F. Da Silva, M. V. Ganduglia-Pirovano, J. Sauer, V. Bayer and G. Kresse, Phys. Rev. B: Condens. Matter Mater. Phys., 2007, 75, 045121.

40 C. Loschen, J. Carrasco, K. M. Neyman and F. Illas, Phys. Rev. B. Condens. Matter Mater. Phys., 2007, 75, 035115.

41 P. R. L. Keating, D. O. Scanlon and G. W. Watson, J. Phys.: Condens. Matter, 2009, 21, 405502.

42 J. R. Gispert, Coordination Chemistry, Wiley-VCH Verlag GmbH \& Co. KGaA, Weinheim, Germany, 2008.

43 D. O. Scanlon, A. Walsh, B. J. Morgan, M. Nolan, J. Fearon and G. W. Watson, J. Phys. Chem. C, 2007, 111(22), 7971-7979.

44 A. A. Sokol, A. Walsh and C. R. A. Catlow, Chem. Phys. Lett., 2010, 492, 44- 48.

45 S. Roy, A. Marimuthu, M. S. Hegde and G. Madras, Appl. Catal., $B, 2007, \mathbf{7 3}, 300-310$.

46 S. Roy and M. S. Hegde, Catal. Commun., 2008, 9, 811-815.

47 S. Roy, M. S. Hegde and G. Madras, Appl. Energy, 2009, 86, $2283-2297$.

48 A. Walsh, J. L. F. Da Silva and S. H. Wei, Phys. Rev. Lett., 2008, 100, 256401. 
49 V. Butler, C. R. A. Catlow, B. E. F. Fender and J. H. Harding, Solid State Ionics, 1983, 8, 109-103.

50 G. Dutta, U. V. Waghmare, T. Baidya, M. S. Hegde, K. R. Priolkar and P. R. Sarode, Chem. Mater., 2006, 18, 3249.

51 G. Dutta, U. V. Waghmare, T. Baidya, M. S. Hegde, K. R. Priolkar and P. R. Sarode, Catal. Lett., 2006, 108, $165-172$.

52 H. F. Wang, X. Q. Gong, Y. L. Guo, Y. Guo, G. Z. Lu and P. Hu, J. Phys. Chem. C, 2009, 113, 10229-10232.
53 Q. W. Wen, H. W. Zhang, Y. W. Song, Q. H. Yang, H. Zhu and J. Q. Xiao, J. Phys.: Condens. Matter, 2007, 19, 246205.

54 Y. Q. Song, H. W. Zhang, Q. Y. Wen, L. Peng and J. Q. Xiao, J. Phys.: Condens. Matter, 2008, 20, 255210.

55 Y. Q. Song, H. W. Zhang, Q. H. Yang, Y. L. Liu, Y. X. Li, L. R. Shah, H. Zhu and J. Q. Xiao, J. Phys.: Condens. Matter, 2009, 21, 125504.

56 V. Ferrari, A. M. Llois and V. Lidosola, J. Phys.: Condens. Matter, 2010, 22, 276002. 